\title{
Nascimento prematuro e o novo coronavírus: Uma revisão integrativa
}

\author{
Premature birth and the new coronavirus: An integrative review \\ Nacimiento prematuro y el nuevo coronavirus: Una revisión integradora
}

Recebido: 19/05/2021 | Revisado: 29/05/2021 | Aceito: 02/06/2021 | Publicado: 17/06/2021

\author{
Jolline Lind \\ ORCID: https://orcid.org/0000-0003-4890-3869 \\ Unimed Curitiba, Brasil \\ E-mail: jolline.lind@unimedcuritiba.com,br \\ Bianca Fontana Aguiar \\ ORCID: https://orcid.org/0000-0001-9054-8245 \\ Unimed Curitiba, Brasil \\ E-mail: bianca.aguiar@unimedcuritiba.com.br \\ Beatriz Böger \\ ORCID: https://orcid.org/0000-0003-0025-2315 \\ Unimed Curitiba, Brasil \\ E-mail: beatriz.boger@unimedcuritiba.com.br \\ Harli Pasquini-Netto \\ ORCID: https://orcid.org/0000-0002-4391-676X \\ Unimed Curitiba, Brasil \\ E-mail: harli.netto@unimedcuritiba.com.br \\ Rafaella Tiepo Borges Abatti \\ ORCID: https://orcid.org/0000-0001-9301-9656 \\ Unimed Curitiba, Brasil \\ E-mail: rafaellaborges@unimedcuritiba.com.br \\ Moacir Pires Ramos \\ ORCID: https://orcid.org/0000-0002-9126-0689 \\ Unimed Curitiba, Brasil \\ E-mail: moacir.ramos@ unimedcuritiba.com.br \\ Jaime Luis Lopes Rocha \\ ORCID: https://orcid.org/0000-0002-3749-6826 \\ Unimed Curitiba, Brasil \\ E-mail: jaimerocha@unimedcuritiba.com.br
}

\begin{abstract}
Resumo
O objetivo principal desta revisão integrativa foi identificar evidências científicas presentes nas publicações sobre a relação do nascimento prematuro com a Covid-19. Como objetivo secundário, verificar outros desfechos clínicos presentes nas gestantes incluídas nesta revisão. Foram utilizados critérios de seleção e descritores controlados para a busca de artigos indexados nas bases de dados Medline/Pubmed e Lilacs em abril de 2021. A seleção final foi constituída por 23 artigos. Os estudos mostram que parto prematuro e sofrimento fetal são possíveis complicações da gravidez de mulheres com Covid-19, foi observado também taxas altas de parto cesárea nesta população. Conclui-se que a Covid-19 em mulheres grávidas tem impactos importantes sobre os resultados perinatais e neonatais. Os resultados mencionados nos estudos podem fornecer uma orientação adicional para melhorar o aconselhamento prénatal das pacientes com infecção por Covid-19 durante a gravidez.
\end{abstract}

Palavras-chave: Gestantes; Infecções por coronavírus; Nascimento prematuro; Revisão.

\begin{abstract}
The main objective of this integrative review was to identify scientific evidence present in publications on the relationship between premature birth and Covid-19. As a secondary objective, to verify other clinical outcomes present in the pregnant women included in this review. Selection criteria and controlled descriptors were used to search for articles indexed in the Medline / Pubmed and Lilacs databases in April 2021. The final selection consisted of 23 articles. Studies show that premature birth and fetal distress are possible complications of pregnancy in women with Covid-19, high rates of cesarean delivery were also observed in this population. It is concluded that Covid-19 in pregnant women has important impacts on perinatal and neonatal outcomes. The results mentioned in the studies may provide additional guidance to improve prenatal counseling for patients with Covid-19 infection during pregnancy.

Keywords: Pregnant women; Coronavirus infections; Premature birth; Review.
\end{abstract}

\section{Resumen}

El principal objetivo de esta revisión integradora fue identificar la evidencia científica presente en las publicaciones sobre la relación entre el nacimiento prematuro y Covid-19. Como objetivo secundario, verificar otros desenlaces 
clínicos presentes en las gestantes incluidas en esta revisión. Se utilizaron criterios de selección y descriptores controlados para la búsqueda de artículos indexados en las bases de datos Medline / Pubmed y Lilacs en abril de 2021. La selección final consistió en 23 artículos. Los estudios muestran que el parto prematuro y el sufrimiento fetal son posibles complicaciones del embarazo en mujeres con Covid-19; también se observaron altas tasas de parto por cesárea en esta población. Se concluye que Covid-19 en mujeres embarazadas tiene impactos importantes en los resultados perinatales y neonatales. Los resultados mencionados en los estudios pueden proporcionar una guía adicional para mejorar el asesoramiento prenatal para pacientes con infección por Covid-19 durante el embarazo.

Palabras clave: Mujeres embarazadas; Infecciones por coronavirus; Nacimiento prematuro; Revisión.

\section{Introdução}

A pandemia de Coronavírus Disease-2019 (Covid-19), a qual se manifestou no final de 2019 em Wuhan, na China e, é causada pelo SARS-CoV-2, disseminou-se por todos os continentes, aumentando de forma expressiva o número de infectados e ocasionando milhares de mortes no mundo (Zhu et al., 2020).

Com relação aos grupos de risco, inicialmente acreditava-se que, da mesma maneira que havia acontecido com a infecção pelo H1N1, em razão das modificações próprias da gestação, a Covid-19 fosse mais grave nas gestantes. Entretanto, os primeiros relatos advindos da China mostravam que esta infecção acometia, com a mesma frequência e gravidade, mulheres grávidas e não grávidas (Chen et al., 2020).

Desse modo, a preocupação inicial teve como foco as gestantes de alto risco, devido a doenças pré-existentes como: hipertensão, diabetes e obesidade, as quais poderiam apresentar pior evolução da Covid-19, de maneira semelhante ao que se observava nas não grávidas (Brasil, 2020).

A literatura tem mostrado maior risco de complicações maternas principalmente no último trimestre da gravidez e no puerpério, inclusive com casos de morte materna (Rasmussen \& Jamieson, 2020). No Brasil, tem-se observado aumento de casos de gestantes infectadas que resultaram em parto prematuro e parto cesárea (Brasil, 2020).

Atualmente, existe o entendimento de que as gestantes e puérperas constituem grupo de risco frente à Covid-19 (Knight et al., 2020; Brasil, 2020). Nesse sentido e devido a importância do assunto, esta revisão integrativa tem por objetivo principal identificar as evidências científicas sobre a relação da Covid-19 com o nascimento prematuro. Como objetivo secundário, verificar outros desfechos clínicos presentes nas gestantes incluídas nesta revisão.

\section{Metodologia}

Trata-se de revisão integrativa, desenvolvida em seis fases: definição da questão de pesquisa, busca na literatura, coleta de dados, análise crítica dos estudos incluídos, discussão dos resultados e apresentação da revisão integrativa (Souza, Silva \& Carvalho, 2010).

Com base na questão de pesquisa: "Existe relação da Covid-19 com nascimentos prematuros?”, foi realizada uma busca de artigos nas bases de dados Medical Literature Analysis and Retrieval System Online (Medline), via PubMed, e Literatura Latino - Americana e do Caribe em Ciências da Saúde (LILACS), ente março de 2020 a 05 de abril de 2021. Os descritores utilizados foram: "Pregnancy"; "Coronavirus Infections", “COVID-19", "Premature Birth” combinados com os operadores booleanos AND e OR.

Posteriormente à busca, foi feita a leitura dos títulos e resumos dos artigos recuperados, dentre os quais, foram selecionados para elegibilidade aqueles que atenderam aos critérios de inclusão que foram: estar em alfabeto latino, a população de estudo serem Gestantes com Covid-19, com pesquisa sobre nascimento prematuro. Foram excluídas publicações que não estavam disponíveis integralmente, cartas, editoriais de periódicos científicos, relatos de apenas um único caso e publicações que não atendiam à temática proposta. Os artigos duplicados foram computados apenas uma vez. 
Para extração dos dados, dois pesquisadores realizaram a leitura dos artigos selecionados na íntegra, com emprego de instrumento de coleta, desenvolvido através de planilhas no Excel ${ }^{\circledR}$ 2010, contendo: identificação do artigo, tema, objetivo, metodologia empregada, nível de evidência, amostra/população do estudo, resultados e conclusões.

Os artigos foram avaliados e classificados quanto ao seu rigor científico conforme as características de cada estudo, utilizando o instrumento do Joanna Briggs Institute (JBI). A instituição preconiza a classificação de estudos de forma piramidal, sendo a base o nível 5 (opinião de especialistas); o nível 4, com os estudos observacionais descritivos; nível 3, observacionais analíticos; nível 2, quase experimentais e nível 1 experimentais. Em cada nível há também subdivisões em letras. Exemplificando, no nível 1 tem-se: 1a, revisões sistemáticas (RS) de estudos controlados e randomizados (ECR); 1b, RS de ECR e outros desenhos; 1c, ECR e 1d pseudos ECRs (Joanna Briggs Institute, 2013).

As estratégias de discussão e interpretação dos resultados, bem como síntese dos dados extraídos são apresentados de forma descritiva.

\section{Resultados}

Foram encontradas 120 publicações (120 nas bases de dados Medline/PubMed e nenhum na LILACS). Desses, após análise dos critérios de inclusão/exclusão e fase de triagem (leitura de título e resumos) permaneceram 60 publicações para leitura na íntegra. Ao final foram selecionados 23 artigos que compuseram a amostra final, todos publicados no ano de 2020 e no idioma inglês.

Em relação ao tipo de estudo, foram identificadas oito revisões sistemáticas (Capobianco et al., 2020; Dubey, Reddy, Manuel \& Dwivedi, 2020; Gao et al., 2020; Matar et al., 2020; Melo \& Araújo, 2020; Novoa et al., 2020; Smith et al., 2020; Yee et al., 2020), um quase-experimento ( Been et al., 2020), cinco coortes prospectivos (Antoun, El Taweel, Ahmed, Patni \& Honest, 2020; Khoury et al., 2020; Oncel et al., 2020; Pirjani et al., 2020; Savasi et al., 2020 , quatro coortes históricas (Sattari et al., 2020; Sentilhes et al., 2020; Yan et al., 2020; Yang et al., 2020), um caso-controle (Li et al., 2020), uma revisão rápida (Mullins, Evans, Viner, O'Brien, \& Morris, 2020), um overview de revisões sistemáticas (Papanou et al., 2020), e duas séries de casos (Remaeus et al., 2020; Turan et al., 2020). A Tabela 1 apresenta as características de cada estudo em relação ao tipo de estudo, objetivos e nível de evidência.

Tabela 1. Dados gerais sobre os artigos incluídos no estudo.

\begin{tabular}{|c|c|c|c|c|}
\hline Autores/Ano & Tipo de estudo & $\begin{array}{l}\text { Número de estudos } \\
\text { incluídos }\end{array}$ & Objetivo & Nível de evidência* \\
\hline $\begin{array}{l}\text { Antoun, El Taweel, } \\
\text { Ahmed, Patni \& } \\
\text { Honest, 2020 }\end{array}$ & Coorte prospectivo & NA & $\begin{array}{l}\text { Estudar o efeito da Covid-19 na } \\
\text { gravidez e nos resultados } \\
\text { neonatais. }\end{array}$ & $3 c$ \\
\hline Been et al., 2020 & Quase experimento & NA & $\begin{array}{l}\text { Estudar o impacto das medidas } \\
\text { de mitigação da Covid- } 19 \\
\text { implementadas na Holanda de } \\
\text { forma gradual em } 9 \text { de março, } \\
15 \text { de março e } 23 \text { de março de } \\
2020 \text {, na incidência de parto } \\
\text { prematuro. }\end{array}$ & $2 \mathrm{c}$ \\
\hline $\begin{array}{l}\text { Capobianco et al., } \\
2020\end{array}$ & $\begin{array}{l}\text { Revisão sistemática e } \\
\text { meta-análise de estudos } \\
\text { observacionais }\end{array}$ & 13 & $\begin{array}{l}\text { Avaliar o risco de complicações } \\
\text { clínicas em mulheres grávidas e } \\
\text { recém-nascidos infectados com } \\
\text { SARS-CoV-2. }\end{array}$ & $2 b$ \\
\hline $\begin{array}{lr}\text { Dubey, } & \text { Reddy, } \\
\text { Manuel \& } & \text { Dwivedi, } \\
2020 & \end{array}$ & $\begin{array}{l}\text { Revisão sistemática e } \\
\text { meta-análise }\end{array}$ & 61 & $\begin{array}{l}\text { Estimar as características } \\
\text { maternas e neonatais adversas } \\
\text { entre mulheres infectadas com } \\
\text { Covid-19. }\end{array}$ & $1 b$ \\
\hline Gao et al., 2020 & $\begin{array}{l}\text { Revisão sistemática e } \\
\text { meta-análise }\end{array}$ & 14 & \begin{tabular}{lll} 
Revisar & as & \multicolumn{2}{c}{ características } \\
clínicas e os desfechos de
\end{tabular} & $1 b$ \\
\hline
\end{tabular}




\begin{tabular}{|c|c|c|c|c|}
\hline & & & $\begin{array}{l}\text { mulheres grávidas com Covid- } \\
19 .\end{array}$ & \\
\hline Khoury et al., 2020 & Coorte prospectivo & NA & $\begin{array}{l}\text { Descrever as características e os } \\
\text { desfechos do nascimento de } \\
\text { grávidas com Covid-19 na } \\
\text { cidade de Nova York. }\end{array}$ & $3 c$ \\
\hline Li et al., 2020 & Caso-controle & NA & $\begin{array}{l}\text { Comparar as características } \\
\text { clínicas e os desfechos maternos } \\
\text { e neonatais de gestantes com e } \\
\text { sem pneumonia por Covid-19. }\end{array}$ & $3 d$ \\
\hline Matar et al., 2020 & $\begin{array}{l}\text { Revisão sistemática e } \\
\text { meta-análise }\end{array}$ & 24 & $\begin{array}{l}\text { Descrever as características } \\
\text { clínicas e os resultados } \\
\text { perinatais da Covid-19 na } \\
\text { gravidez. }\end{array}$ & $1 \mathrm{~b}$ \\
\hline Melo \& Araújo, 2020 & $\begin{array}{l}\text { Revisão sistemática e } \\
\text { meta-análise }\end{array}$ & 38 & $\begin{array}{ll}\begin{array}{l}\text { Analisar se a Covid-19 em } \\
\text { mulheres }\end{array} & \text { grávidas está } \\
\text { relacionada } & \text { ao nascimento } \\
\text { prematuro. } & \end{array}$ & $1 \mathrm{~b}$ \\
\hline $\begin{array}{l}\text { Mullins, Evans, } \\
\text { Viner, O'Brien, \& } \\
\text { Morris, 2020 }\end{array}$ & Revisão rápida & 21 & $\begin{array}{l}\text { Orientar a elaboração } \\
\text { diretrizes } \\
\text { Covid-19 na gravidez. }\end{array}$ & $4 a$ \\
\hline Novoa et al., 2020 & Revisão sistemática & 37 & $\begin{array}{l}\text { Descrever os desfechos } \\
\text { maternos e perinatais de } \\
\text { gestantes positivas para Covid- } \\
19 .\end{array}$ & $1 b$ \\
\hline Oncel et al., 2020 & Coorte multicêntrico & NA & $\begin{array}{l}\text { Avaliar a epidemiologia e } \\
\text { características clínicas de } \\
\text { recém-nascidos de mulheres } \\
\text { infectadas com Covid-19. }\end{array}$ & $3 c$ \\
\hline Papanou et al., 2020 & $\begin{array}{l}\text { Overview de revisões } \\
\text { sistemáticas }\end{array}$ & 39 & $\begin{array}{l}\text { Descrever o quadro obstétrico- } \\
\text { perinatal e os desfechos de } \\
\text { mulheres grávidas infectadas } \\
\text { por Covid-19. }\end{array}$ & 1a \\
\hline Pirjani et al., 2020 & Coorte prospectivo & NA & $\begin{array}{l}\text { Comparar as consequências } \\
\text { maternas e fetais da Covid-19 } \\
\text { em gestações infectadas e não } \\
\text { infectadas. }\end{array}$ & $3 c$ \\
\hline Remaeus et al., 2020 & $\begin{array}{l}\text { Série de casos } \\
\text { retrospectivos }\end{array}$ & NA & $\begin{array}{l}\text { Descrever as características } \\
\text { clínicas em } 67 \text { mulheres com } \\
\text { SARS-CoV-2 positivas e seus } \\
68 \text { recém-nascidos, e relatar } \\
\text { desfechos maternos e neonatais } \\
\text { de curto prazo. }\end{array}$ & $4 \mathrm{c}$ \\
\hline Sattari et al., 2020 & Coorte histórica & NA & $\begin{array}{l}\text { Avaliar o curso clínico e os } \\
\text { fatores de risco de mulheres } \\
\text { grávidas com diagnóstico de } \\
\text { Covid-19 na província de } \\
\text { Hamadan, oeste do Irã. }\end{array}$ & $3 c$ \\
\hline Savasi et al., 2020 & $\begin{array}{l}\text { Coorte prospectivo } \\
\text { multicêntrico }\end{array}$ & NA & $\begin{array}{l}\text { Investigar a evolução clínica da } \\
\text { Covid-19 em gestantes } \\
\text { hospitalizadas e os potenciais } \\
\text { fatores associados a desfechos } \\
\text { maternos graves. }\end{array}$ & $3 c$ \\
\hline Sentilhes et al., 2020 & $\begin{array}{l}\text { Coorte histórica de } \\
\text { centro único }\end{array}$ & NA & $\begin{array}{l}\text { Relatar as características } \\
\text { maternas e desfechos de } \\
\text { mulheres grávidas com Covid- } \\
19 .\end{array}$ & $3 c$ \\
\hline Smith et al., 2020 & Revisão sistemática & 9 & $\begin{array}{l}\text { Avaliar sistematicamente a } \\
\text { literatura e relatar os resultados } \\
\text { maternos e neonatais associados } \\
\text { a Covid-19. }\end{array}$ & $1 \mathrm{~b}$ \\
\hline Turan et al., 2020 & Série de casos & NA & 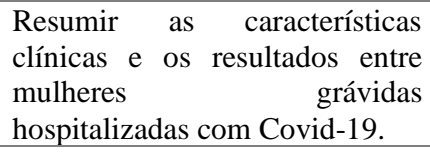 & $4 \mathrm{c}$ \\
\hline Yan et al., 2020 & Coorte histórica & NA & Avaliar as características & $3 c$ \\
\hline
\end{tabular}




\begin{tabular}{|c|c|c|c|c|}
\hline & & & Aguda Grave por coronavírus. & \\
\hline Yang et al., 2020 & Coorte histórica & NA & $\begin{array}{l}\text { Avaliar as associações entre o } \\
\text { diagnóstico materno de Covid- } \\
19 \text { e os resultados adversos do } \\
\text { nascimento. }\end{array}$ & $3 c$ \\
\hline Yee et al., 2020 & $\begin{array}{l}\text { Revisão sistemática e } \\
\text { meta-análise }\end{array}$ & 11 & $\begin{array}{l}\text { Avaliar o impacto da Covid-19 } \\
\text { em mulheres grávidas. }\end{array}$ & $1 b$ \\
\hline
\end{tabular}

Nota. Covid-19: doença do coronavírus de 2019; NA: Não se aplica; * Joanna Briggs Institute. Fonte: Autores.

Referente ao local de pesquisa, 17,4\% ( $\mathrm{n}=4$ ) deles ocorreram na China (Gao et al., 2020; Li et al., 2020; Yan et al., 2020; Yang et al., 2020), 13,2\% (n=3) deles ocorreram respectivamente nos Estados Unidos da América (Dubey, Reddy, Manuel \& Dwivedi, 2020; Khoury et al., 2020; Matar et al., 2020) e na Inglaterra (Antoun, El Taweel, Ahmed, Patni \& Honest, 2020; Mullins, Evans, Viner, O'Brien, \& Morris, 2020; Turan et al., 2020). Irã (Pirjani et al., 2020; Sattari et al., 2020), Brasil (Melo \& Araújo, 2020; Smith et al., 2020), e Itália (Capobianco et al., 2020; Savasi et al., 2020), tiveram apenas dois (8,7\%) artigos cada. Ainda, Holanda (Been et al., 2020), Peru (Novoa et al., 2020), Turquia (Oncel et al., 2020), Grécia (Papanou et al., 2020), Suécia (Remaeus et al., 2020), França (Sentilhes et al., 2020), Coreia (Yee et.al, 2020), tiveram um artigo $(4,3 \%)$ cada.

O número de gestantes avaliadas nos estudos apresentou variabilidade, de 23 em um coorte prospectivo (Antoun, El Taweel, Ahmed, Patni \& Honest, 2020) a 11.078 (Yang et al., 2020). Onze estudos (47,83\%) incluídos nessa revisão e que apresentaram em seus resultados o tipo de parto, relatam taxas altas de parto cesárea (acima de 70\%) (Antoun, El Taweel, Ahmed, Patni \& Honest, 2020; Capobianco et al., 2020; Dubey, Reddy, Manuel \& Dwivedi, 2020; Khoury et al., 2020; Matar et al., 2020; Li et al., 2020; Mullins, Evans, Viner, O'Brien, \& Morris, 2020; Oncel et al., 2020; Smith et al., 2020; Turan et al., 2020; Yan et al., 2020).

Oito estudos (34,78\%) apontam que taxas mais altas de parto prematuro são encontradas em gestantes adoecidas pela Covid-19 (Antoun, El Taweel, Ahmed, Patni \& Honest, 2020; Gao et al., 2020; Khoury et al., 2020; Matar et al., 2020; Papanou et al., 2020; Sentilhes et al., 2020; Smith et al., 2020; Turan et al., 2020). Um estudo (4,35\%) demonstra que em comparação com mulheres grávidas sem Covid-19, as com diagnóstico confirmado tiveram um risco aumentado de parto prematuro (Yang et al., 2020). Três estudos (13,04\%) não encontraram associação significativa entre a infecção por Covid-19 e nascimento prematuro (Melo \& Araújo, 2020; Pirjani et al., 2020; Yan et al., 2020). Os principais resultados e conclusões dos estudos incluídos são apresentados na Tabela 2.

Tabela 2. Principais resultados e conclusões das evidências incluídas na revisão.

\begin{tabular}{|c|c|c|c|}
\hline Autores/Ano & $\begin{array}{c}\text { Número de grávidas } \\
\text { estudadas ou } \\
\text { nascimentos }\end{array}$ & Principais resultados & Conclusões \\
\hline 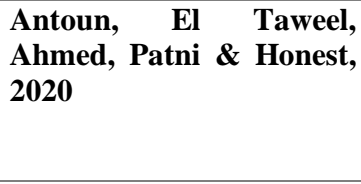 & 23 gestantes & $\begin{array}{l}\text { Parto prematuro: Sete }(36,8 \%) \text {; } \\
\text { Cesárea: } 16(84 \%) \text {. }\end{array}$ & $\begin{array}{l}\text { A Covid-19 está associada a alta } \\
\text { prevalência de parto prematuro, pré- } \\
\text { eclâmpsia e parto cesárea em } \\
\text { comparação com gestações não } \\
\text { Covid-19. }\end{array}$ \\
\hline Been et al., 2020 & 1.599.547 nascimentos & $\begin{array}{l}\text { Reduções consistentes na } \\
\text { incidência de nascimento } \\
\text { prematuro foram observadas em } \\
\text { várias janelas de tempo em torno } \\
\text { de } 9 \text { de março }( \pm 2 \text { meses } \\
{[\mathrm{n}=531.823] \text { razão de chances }} \\
{[\mathrm{OR}] 0,77, \text { IC } 95 \% 0,66-0,91, \mathrm{p}}\end{array}$ & $\begin{array}{l}\text { Medidas de mitigação da Covid- } 19 \\
\text { foram associadas a uma redução } \\
\text { substancial na incidência de partos } \\
\text { prematuros nos meses seguintes. Há } \\
\text { necessidade de explorar os } \\
\text { mecanismos subjacentes. }\end{array}$ \\
\hline
\end{tabular}


$=0,0026 ; \pm 3$ meses $[\mathrm{n}=796.531]$

OR 0,85 , IC $95 \%$ : $0,73-0,98$,

$\mathrm{p}=0,028 ; \pm 4$ meses $[\mathrm{n}=1.066 .872]$

OR 0,84 , IC $95 \%$ : $0,73-0,97, \mathrm{p}=$ $0,023)$

Capobianco et al., $2020 \quad 114$ gestantes $\quad$ Complicações maternas: $45 \%$ (IC 95\%: $24,0 \%-67,0 \%)$;

Cesárea: $88 \%$ (IC 95\%: 82,0\%$94,0 \%)$

Internadas em Unidade de Terapia Intensiva: $<20 \%$;

Bebês prematuros: $23 \%$ (IC 95\%:

$11,0 \%-39,0 \%)$.

\section{Dubey, Reddy, Manuel \& \\ Dwivedi, 2020}

Gao et al., $2020 \quad 236$ gestantes

790 gestantes

\section{Cesárea: $72 \%$;}

Parto prematuro: $23 \%$;

Baixo peso ao nascer: $7 \%$; E eventos adversos: $27 \%$.

Trabalho de parto prematuro:

(23\%; IC 95\%, 0, 14-0,32);

Caso grave ou morte: (12\%; IC $95 \%, 0,03-0,20)$.

Cesárea: $(65 \%$; IC $95 \%, 0,42-$ 0,87).

Khoury et al., 2020

241 gestantes

Taxa de nascimentos pré-termo únicos: $14,6 \%$;

Cesárea: $91,7 \%$ nos casos críticos. Internação na Unidade de Terapia Intensiva: $7,1 \%$;

Nove $(3,7 \%)$ foram intubadas durante a internação do parto.

Li et al., 2020

Matar et al., 2020

136 gestantes

\section{Parto normal: 2 ;}

Cesárea: 32 (94,12\%);

Pneumonia por Covid-19: três $(18,8 \%)$;

Parto prematuro devido complicações maternas: três $(16,7 \%)$.

Taxa de partos prematuros: $37,7 \%$; Cesárea: $76 \%$;

Morte materna de Covid-19: 1; Mortes fetais de Covid-19: 2.

\begin{tabular}{ll}
\hline Melo \& Araújo, 2020 & Covid-19 versus parto prematuro: \\
& \\
& \\
& $\left.=0,06 ; \mathrm{I}^{2}=0 \%\right)$
\end{tabular}

Mullins, Evans, Viner,

O'Brien, \& Morris, 2020

32 gestantes

Morte materna de Covid-19: 0.

Cesárea: 27 (84,37\%);

Partos vaginais: 2 ;

Partos prematuros: 15 (47\%);

Natimorto: 1;

Morte neonatal:1.
Novoa et al., 2020

322 gestantes

Cesárea: 99 (50,8\%);

Parto vaginal: 64 (32,8\%);

Parto prematuro: 37 (18,9\%);

Cuidados em Unidade de Terapia Intensiva: Trinta pacientes $(10,3 \%)$

Morte: Uma (0,3\%);

SARS-CoV-2 estava ausente no leite materno, líquido amniótico, placenta ou sangue do cordão umbilical.

Oncel et al., 2020

125 gestantes
Sugere-se um alto índice de complicações maternas e neonatais em indivíduos infectados. No entanto, os resultados destacam um baixo risco de infecção neonatal.

O rastreamento regular e a detecção precoce da Covid-19 em mulheres grávidas podem fornecer resultados mais favoráveis.

A taxa de trabalho de parto prematuro é maior entre as grávidas com Covid19 do que entre mulheres grávidas sem Covid-19.

A gravidade da doença foi associada a maiores taxas de cesárea e parto prematuro.

Não foram observadas complicações maternas e neonatais graves em gestantes com pneumonia por Covid19 que tiveram parto vaginal ou cesárea.

O quadro clínico em mulheres grávidas com Covid-19 não diferiu da população não grávida; porém, as taxas de partos prematuros e cesáreas são consideravelmente maiores do que as médias internacionais.

A meta-análise não mostrou associação significativa entre Covid19 e parto prematuro, no entanto, as evidências são muito incertas.

Recomenda-se que o tipo de parto seja determinado principalmente por indicação obstétrica e não se recomenda a separação de rotina das mães afetadas e seus recém-nascidos.

Nascimento prematuro iatrogênico é o principal resultado obstétrico adverso. Os dados atuais não suportam a transmissão vertical no terceiro trimestre.

Covid-19 em mulheres grávidas tem impactos importantes nos resultados perinatais e neonatais.
Taxa de prematuridade: $26,4 \%$;

Taxa de bebê com baixo peso ao nascer: $12,8 \%$; 
Cuidados em Unidade de Terapia Intensiva: Oito $(6,4 \%)$; Mortes: seis $(4,8 \%)$.

Papanou et al., 2020 NR Taxa de parto cesárea: 52,3 -
$95,8 \%$

Taxa de partos vaginais prematuros: $14,3-63,8 \%$.

\section{Pirjani et al., 2020}

199 gestantes

Cesárea: 105 (52,76\%);
Tipo de parto em gestantes infectadas e não infectadas com Covid-19 (RR: 1,31, IC 95\%: 1,04$1,65, \quad \mathrm{p}=0,024)$. Nenhuma associação significativa foi encontrada entre a infecção por Covid-19 e nascimento prematuro (RR: 1,16 , IC 95\%: 0,54- 2,48, p= $0,689)$, baixo peso ao nascer (RR: 1,13, IC 95\%: 0,55 - 2,31, $\mathrm{p}=0,723)$, diabetes gestacional (RR: 1,67, IC 95\%: 0,81-3,42, p = 0,160 ), pré-eclâmpsia (RR: 2,02, IC 95\%: 0,42-6,78, $\mathrm{p}=0,315)$, restrição de crescimento intrauterino (RR: 0,16, IC 95\%: $0,02-1,86, \quad \mathrm{p}=0,145)$, ruptura prematura da membrana (RR: 0,19 , IC 95\%: 0,02-2,20, $\mathrm{p}=0,186$ ), natimorto (RR: 1,41 , IC 95\%: 0,08-18,37, $\mathrm{p}=0,614)$, hemorragia pós-parto (RR: 1,84 , IC 95\%: 0,39$8,63, \mathrm{p}=0,185$ ), admissão na unidade de terapia intensiva neonatal (RR: 1,84, IC 95\%: 0,77- 4,39, $p=0,168)$, sepse neonatal (RR: 0,84, IC 95\%: 0,48$1,48, \mathrm{p}=0,568)$.

Remaeus et al., 2020

67 gestantes

Foram encontradas taxas aumentadas de cesárea e de nascimentos prematuros. A probabilidade de transmissão vertical não pode ser excluída. Mais estudos originais em mulheres de todos os trimestres são necessários.

Embora os resultados da gravidez e neonatais não tenham sido significativamente diferentes, a necessidade de cuidados na Unidade de Terapia Intensiva para mulheres grávidas com Covid-19 foi significativamente maior em comparação com aquelas sem Covid19.

Nesta série de casos poucas mulheres Cuidados em Unidade de Terapia apresentaram Covid-19 grave e a Intensiva: 4 (6\%);

Os partos prematuros foram em sua maioria com indicação médica, incluindo duas mulheres que tiveram parto prematuro por doença grave, correspondendo a $15 \%$ dos partos prematuros.

\begin{tabular}{|c|c|c|c|}
\hline Sattari et al., 2020 & 50 gestantes & $\begin{array}{l}\text { Parto prematuro: } 29 \% \text {; } \\
\text { Parto normal: } 28 \% \text {; } \\
\text { Cesárea: } 20 \%\end{array}$ & $\begin{array}{l}\text { O diagnóstico precoce da Covid-19 é } \\
\text { essencial em mulheres grávidas. }\end{array}$ \\
\hline Savasi et al., 2020 & 77 gestantes & $\begin{array}{l}\text { O parto prematuro: } 12 \% \text {; } \\
\text { Cuidados em Unidade de Terapia } \\
\text { Intensiva Neonatal: } 9 .\end{array}$ & $\begin{array}{l}\text { No estudo uma em cada cinco } \\
\text { mulheres hospitalizadas com infecção } \\
\text { por Covid- } 19 \text { teve parto urgente por } \\
\text { comprometimento respiratório ou } \\
\text { internamento na Unidade de Terapia } \\
\text { Intensiva. }\end{array}$ \\
\hline Sentilhes et al., 2020 & 54 gestantes & $\begin{array}{l}\text { Aborto espontâneo: } 1 \text {; } \\
\text { Cesárea: } 9 \text {; } \\
\text { Parto vaginal: } 12 \text {; } \\
\text { Parto prematuro: } 5 \text { de } 21 \text { mulheres } \\
(23,8 \%): 3(14,3 \%) \text { antes de } 32 \\
\text { semanas de gestação e } 2(9,5 \%) \\
\text { antes de } 28 \text { semanas de gestação. }\end{array}$ & $\begin{array}{l}\text { A Covid-19 na gravidez foi associada } \\
\text { com morbidade materna e nascimento } \\
\text { prematuro. Sua associação com } \\
\text { outros fatores de risco bem } \\
\text { conhecidos para morbidade materna } \\
\text { grave em mulheres grávidas sem } \\
\text { infecção, incluindo idade materna } \\
\text { acima de } 35 \text { anos, sobrepeso e } \\
\text { obesidade, sugere que mais estudos }\end{array}$ \\
\hline
\end{tabular}




\begin{tabular}{|c|c|c|c|}
\hline & & & nessas mulheres. \\
\hline Smith et al., 2020 & 92 gestantes & $\begin{array}{l}\text { Mortalidade materna: } 0 \% \text {; } \\
\text { Cuidados intensivos e ventilação: } \\
\text { 1; } \\
\text { Taxa de partos prematuros: } 63,8 \% \text {; } \\
\text { Sofrimento fetal: } 61,1 \% \text {; } \\
\text { Cesárea: } 80 \% \text {. }\end{array}$ & $\begin{array}{l}\text { A incidência de nascimentos } \\
\text { prematuros, baixo peso ao nascer, } \\
\text { cesárea, admissão em Unidade de } \\
\text { Terapia Intensiva Neonatal parecem } \\
\text { maiores do que a população geral. }\end{array}$ \\
\hline Turan et al., 2020 & 637gestantes & $\begin{array}{l}\text { Taxa de nascimento prematuro } \\
\text { geral: } 33,7 \% \text {; } \\
\text { Taxa de nascimento prematuro em } \\
\text { mulheres com Covid-19 leve: } \\
15,35 \% \text {; } \\
\text { Cesárea: } 84,1 \% \text {; } \\
\text { Idade avaçada, obesidade, } \\
\text { diabetes mellitus e aumento } \\
\text { Dímero D sérico e interleucina-6 } \\
\text { foram preditivos de resultados } \\
\text { ruins. }\end{array}$ & $\begin{array}{l}\text { Gestação avançada, idade materna, } \\
\text { obesidade, diabetes mellitus e uma } \\
\text { combinação de dímero D elevado e } \\
\text { níveis de interleucina- } 6 \text { são preditivos } \\
\text { de resultados ruins em Covid-19. A } \\
\text { taxa de parto prematuro iatrogênico e } \\
\text { parto cesárea é alta. A transmissão } \\
\text { vertical pode ser possível, mas não foi } \\
\text { comprovada. }\end{array}$ \\
\hline Yan et al., 2020 & 116 gestantes & $\begin{array}{l}\text { Taxa de nascimento prematuro } \\
\text { espontâneo: } 6,1 \%(6 / 99) ; \\
\text { Cesárea: } 85,9 \% \text {. }\end{array}$ & $\begin{array}{l}\text { Síndrome respiratória aguda grave } \\
\text { por Covid-19 e a infecção durante a } \\
\text { gravidez não está associada a um } \\
\text { risco aumentado de aborto } \\
\text { espontâneo e parto prematuro } \\
\text { espontâneo. }\end{array}$ \\
\hline Yang et al., 2020 & 11.078 gestantes & $\begin{array}{l}\text { Parto prematuro em mulheres } \\
\text { grávidas sem Covid-19 versus } \\
\text { mulheres grávidas com Covid-19: } \\
\text { (OR 3,34, IC 95\% 1,60-7,00); } \\
\text { Cesárea em grávidas sem Covid-19 } \\
\text { versus grávidas com Covid-19 } \\
\text { versus: (OR 3,63, IC 95\% 1,95- } \\
6,76) \text {. } \\
\text { Não houve diferença estatística no } \\
\text { baixo peso ao nascer, asfixia } \\
\text { neonatal entre as mães com e sem } \\
\text { Covid-19. }\end{array}$ & $\begin{array}{l}\text { É importante monitorar os efeitos a } \\
\text { longo prazo da infecção por SARS- } \\
\text { CoV-2 na saúde em mulheres } \\
\text { grávidas e seus filhos. }\end{array}$ \\
\hline Yee et al., 2020 & 9.032 gestantes & $\begin{array}{l}\text { Parto prematuro: } 30 \% \text {; } \\
\text { Peso médio ao nascer: } 2,855 \mathrm{Kg} \text {. } \\
\text { Morte fetal: } 2 \% \text {; } \\
\text { Detecção de SARS-CoV-2: } 2 \% \text {. }\end{array}$ & $\begin{array}{l}\text { A revisão poderá servir como uma } \\
\text { base ideal para futuras considerações } \\
\text { no tratamento da Covid-19 em } \\
\text { mulheres grávidas. }\end{array}$ \\
\hline
\end{tabular}

Nota. Covid-19: doença do coronavírus de 2019; NR: não reportado; RR: risco relativo; OR: razão de chances; IC 95\%: intervalo de confiança de $95 \%$; $\mathrm{I}^{2}$ : índice de heterogeneidade. Fonte: Autores.

\section{Discussão}

\subsection{Taxas de nascimento prematuro}

O nascimento prematuro é o principal contribuinte para a mortalidade e morbidade na primeira infância (Chawanpaiboon et al., 2014). Os sobreviventes cursam com risco de consequências negativas de longo prazo, incluindo desenvolvimento cognitivo e motor adverso, (Twilhaar et al., 2019; Vollmer \& Stålnacke, 2019) problemas de saúde mental e comportamental, (Franz et al., 2018) e desordens respiratória (Been et al., 2014). Globalmente, a incidência de nascimentos prematuros está em alta, (Chawanpaiboon et al., 2014) e as opções atuais para prevenção são muito limitadas (Matei, Sacone, Vogel \& Armson, 2019). Os estudos analisados demonstraram variabilidade nas taxas de nascimento prematuro. Há taxas de 14,6\% (Khoury et al., 2020) a 84\% (Antoun et al., 2020). Segundo Novoa et al (2020) o nascimento prematuro iatrogênico é o principal resultado obstétrico adverso. Cabe destacar, que no Brasil ocorrem em média, aproximadamente 340 mil partos 
prematuros por ano, (Brasil, 2020) e consequentemente devido a Covid-19, há possibilidade de se registrar um aumento deste índice.

As intervenções de saúde pública devem ser cuidadosamente implementadas e adaptadas a esses importantes grupos suscetíveis para reduzir a incidência de infecção e, então, o risco de grandes complicações. Um acompanhamento regular e intensivo é necessário para detectar precocemente a ocorrência de condições clínicas indesejáveis (Capobianco et al., 2020).

\subsection{Características clínicas, sinais e sintomas e outros desfechos}

As características clínicas de grávidas com pneumonia por Covid-19 são semelhantes às de mulheres não grávidas com a mesma patologia. Atualmente, não há evidências de que grávidas com Covid-19 são mais propensas a ter pneumonia grave do que pacientes não grávidas (Yan et al., 2020). Ao contrário do que se viu em 2009, com a pandemia de influenza A, em que as gestantes que contraíram o vírus H1N1, estavam entre os casos mais graves da doença, com maior necessidade de hospitalização e alto risco de mortalidade (Siston et al., 2010).

Com relação a sintomatologia, estudo de coorte retrospectivo refere que os sintomas mais comuns em mulheres grávidas com Covid-19 foram febre, tosse e dificuldade respiratória (Sattari et al., 2020). Coorte prospectivo menciona que febre e dispneia na admissão eram sintomas significativamente associados a subsequente deterioração respiratória. $\mathrm{O}$ estudo salienta ainda que o aumento do Índice de Massa Corpórea (IMC) foi um fator de risco significativo para o agravamento da Covid-19 (Savari et al., 2020).

A prematuridade é citada nos estudos como o resultado perinatal adverso mais prevalente (Savari et al., 2020; Sattari et al., 2020; Novoa et al., 2020). Com relação a morbidade e mortalidade maternas, a literatura destaca que há semelhança aos dados relatados para mulheres não grávidas (Novoa et al., 2020). Revisão sistemática ressalta que a admissão em Unidade de Terapia Intensiva Neonatal (UTIN) parece maior do que na população em geral (Smith et al., 2020).

Série de casos conclui que à medida que o entendimento coletivo cresce, acredita-se que os obstetras se tornarão menos intervencionistas, especialmente em gestações prematuras, ao tratar a Covid-19 leve, reduzindo assim a carga de morbidade neonatal iatrogênica. Os dados suportam achados previamente documentados de desfechos maternos tranquilizadores para infecção leve por Covid-19 e, com desfechos maternos e fetais ruins entre aquelas com doença grave ou crítica, predisposta por obesidade, diabetes mellitus, idade materna avançada e gravidez avançada. Ressalta-se que esse conhecimento deve ser base para aconselhar gestantes com diagnóstico de Covid-19 (Turan et al., 2020). Salienta-se que as drogas recentemente aprovadas para redução de desfechos graves não foram estudadas em gestantes, como por exemplo os anticorpos monoclonais.

\subsection{Tipo de parto}

Estudo de coorte de base populacional em Wuhan mostra que a infecção por SARS-CoV-2 durante o final da gravidez está associado ao aumento de risco de parto prematuro iatrogênico e realização de cesárea (Yang et al., 2020). Meta-análise corrobora com esse dado e demonstra que em comparação com a população geral de grávidas, mulheres infectadas correm um risco maior de cesárea (taxa de 76,3\%; IC de 95\%, 0,658 - 0,842; I²=10,24\%) e nascimento prematuro (taxa de 37,7\%; IC de $95 \%, 0,269-0,500 ; \mathrm{I}^{2}=10,77 \%$ ) (Matar et al., 2020).

Em contrapartida, um estudo quase-experimental demonstrou que a introdução nacional de medidas de mitigação da Covid-19 na Holanda foi associada a uma redução considerável em nascimentos prematuros (Been et al., 2020). Já em uma revisão sistemática com metanálise também referente a investigação de prematuridade em mulheres grávidas com Covid-19 concluiu que a infecção pode não estar associado à ocorrência de partos prematuros ( $\mathrm{OR}=2,25$; IC 95\%: 0,96-5,31; $\mathrm{p}=0,06$; $\mathrm{I}^{2}=0 \%$ ), no entanto, as evidências até o momento são muito incertas (Melo \& Araújo, 2020). 


\subsection{Transmissão vertical}

Outra metanálise demonstra que grávidas com Covid-19 têm características clínicas e resultados semelhantes aos encontrados na população não grávida e parece haver pouca evidência de transmissão vertical (Matar et al., 2020). Revisão sistemática e estudos de coorte retrospectivos corroboram com a consideração acima e reforçam que não há evidências convincentes de transmissão vertical, pelo menos durante o terceiro trimestre de gravidez (Novoa et al, 2020; Yan et al., 2020; Yang et al., 2020). Portanto, não há risco aumentado de má formação e de óbito intrauterino.

Pesquisas estão em andamento com o objetivo de responder a algumas das perguntas em relação ao risco de infecção congênita, manejo intraparto e tipo de parto (Yan et al., 2020).

\subsection{Necessidade de realização de mais estudos}

Infelizmente, ainda não há evidências suficientes sobre o vírus na gravidez (Pirjani et al., 2020). Mortalidade materna, maiores taxas de parto prematuro e cesárea, risco de transmissão vertical, e o possível papel da gravidade da doença materna nos resultados devem ser avaliados em estudos futuros (Oncel et al., 2020). Outros estudos originais com mulheres de todos os trimestres e mais longos períodos de acompanhamento são necessários (Papanou et al., 2020). Além disso, alguns estudos destacam a necessidade de rastreamento efetivo na admissão (Li et al., 2020; Dubey, Reddy, Manuel \& Dwivedi, 2020). Assim como, estudos relacionados ao uso de vacinas na prevenção da doença, principalmente nas formas graves. Como também a realização de estudos sobre risco, gravidade e incidência de doença tromboembólica, bem como prevenção.

\subsection{Limitações do estudo}

Nosso estudo apresentou como limitações o não esgotamento da literatura sobre o tema e o fato da Covid-19 ainda ser uma doença muito recente e muitos aspectos da patologia serem desconhecidos. No entanto, acredita-se que os artigos incluídos nessa revisão tenham importante representatividade da produção sobre a relação da Covid-19 com o nascimento prematuro, uma vez que os estudos incluídos são de 2020 e foram publicados em revistas de impacto internacional, amplamente utilizadas para divulgação de resultados de pesquisa científica.

\section{Conclusão}

Os estudos incluídos nessa revisão mostram que parto prematuro e sofrimento fetal são possíveis complicações da gravidez de mulheres com Covid-19, mas a transmissão vertical, parece ser rara. Foram observadas também taxas altas de cesárea. Os resultados mencionados podem fornecer uma orientação adicional para melhorar o aconselhamento pré-natal das pacientes com infecção por Covid-19 durante a gravidez. Melhor monitoramento e acompanhamento das gestantes, assim como estudos futuros de vigilância são necessários para avaliar as consequências adversas da infecção em recém-nascidos.

\section{Agradecimentos}

Os autores gostariam de agradecer à Unimed Curitiba por seu apoio ao desenvolvimento de pesquisa e inovação.

\section{Referências}

Antoun, L., El Taweel, N., Ahmed, I., Patni, S., \& Honest, H. (2020). Maternal COVID-19 infection, clinical characteristics, pregnancy, and neonatal outcome: A prospective cohort study. European Journal of Obstetrics \& Gynecology and Reproductive Biology, 252, $559-562$.

Been, J. V., Lugtenberg, M. J., Smets, E., van Schayck, C. P., Kramer, B. W., Mommers, M., \& Sheikh, A. (2014). Preterm birth and childhood wheezing disorders: a systematic review and meta-analysis. PLoS Med, 11(1), e1001596. 
Been, J. V., Ochoa, L. B., Bertens, L. C., Schoenmakers, S., Steegers, E. A., \& Reiss, I. K. (2020). Impact of COVID-19 mitigation measures on the incidence of preterm birth: a national quasi-experimental study. The Lancet Public Health, 5(11), e604-e611.

Brasil. Ministério da Saúde. Manual de recomendações para a assistência à gestante e puérpera frente à pandemia de Covid-19.

Brasil. Ministério da Saúde. Governo Federal investe R\$ 335 milhões para ampliar o cuidado e prevenção à prematuridade.

Capobianco, G., Saderi, L., Aliberti, S., Mondoni, M., Piana, A., Dessole, F., \& Sotgiu, G. (2020). COVID-19 in pregnant women: A systematic review and meta-analysis. European Journal of Obstetrics \& Gynecology and Reproductive Biology, 252, 543-558.

Chawanpaiboon, S., Vogel, J. P., Moller, A. B., Lumbiganon, P., Petzold, M., Hogan, D., \& Gülmezoglu, A. M. (2019). Global, regional, and national estimates of levels of preterm birth in 2014: a systematic review and modelling analysis. The Lancet Global Health, 7(1), e37-e46.

Chen, N., Zhou, M., Dong, X., Qu, J., Gong, F., Han, Y., \& Zhang, L. (2020). Epidemiological and clinical characteristics of 99 cases of 2019 novel coronavirus pneumonia in Wuhan, China: a descriptive study. The lancet, 395(10223), 507-513.

Dubey, P., Reddy, S., Manuel, S., \& Dwivedi, A. K. (2020). Maternal and neonatal characteristics and outcomes among COVID-19 infected women: An updated systematic review and meta-analysis. European Journal of Obstetrics \& Gynecology and Reproductive Biology.

Franz, A. P., Bolat, G. U., Bolat, H., Matijasevich, A., Santos, I. S., Silveira, R. C., ... \& Moreira-Maia, C. R. (2018). Attention-deficit/hyperactivity disorder and very preterm/very low birth weight: a meta-analysis. Pediatrics, 141(1).

Gao, Y. J., Ye, L., Zhang, J. S., Yin, Y. X., Liu, M., Yu, H. B., \& Zhou, R. (2020). Clinical features and outcomes of pregnant women with COVID-19: a systematic review and meta-analysis. BMC infectious diseases, 20(1), 1-11.

Joanna Briggs Institute. (2013). JBI levels of evidence. Joanna Briggs Institute [Internet]. Adelaide: The Joanna Briggs Institute.

Khoury, R., Bernstein, P. S., Debolt, C., Stone, J., Sutton, D. M., Simpson, L. L., \& Dolan, S. M. (2020). Characteristics and outcomes of 241 births to women with severe acute respiratory syndrome coronavirus 2 (SARS-CoV-2) infection at five New York City medical centers. Obstetrics \& Gynecology, 136(2), 273282 .

Knight, M., Bunch, K., Vousden, N., Morris, E., Simpson, N., Gale, C., \& Kurinczuk, J. J. (2020). Characteristics and outcomes of pregnant women admitted to hospital with confirmed SARS-CoV-2 infection in UK: national population based cohort study. bmj, 369.

Li, N., Han, L., Peng, M., Lv, Y., Ouyang, Y., Liu, K., \& Yang, L. (2020). Maternal and neonatal outcomes of pregnant women with coronavirus disease 2019 (COVID-19) pneumonia: a case-control study. Clinical infectious diseases, 71(16), 2035-2041.

Matar, R., Alrahmani, L., Monzer, N., Debiane, L. G., Berbari, E., Fares, J., ... \& Murad, M. H. (2021). Clinical presentation and outcomes of pregnant women with coronavirus disease 2019: a systematic review and meta-analysis. Clinical Infectious Diseases, 72(3), $521-533$.

Matei, A., Saccone, G., Vogel, J. P., \& Armson, A. B. (2019). Primary and secondary prevention of preterm birth: a review of systematic reviews and ongoing randomized controlled trials. European Journal of Obstetrics \& Gynecology and Reproductive Biology, 236, $224-239$.

Melo, G. C. D., \& Araújo, K. C. G. M. D. (2020). COVID-19 infection in pregnant women, preterm delivery, birth weight, and vertical transmission: a systematic review and meta-analysis. Cadernos de saude publica, 36, e00087320.

Mullins, E., Evans, D., Viner, R. M., O'Brien, P., \& Morris, E. (2020). Coronavirus in pregnancy and delivery: rapid review. Ultrasound in Obstetrics \& Gynecology, 55(5), 586-592.

Novoa, R. H., Quintana, W., Llancarí, P., Urbina-Quispe, K., Guevara-Ríos, E., \& Ventura, W. (2020). Maternal clinical characteristics and perinatal outcomes among pregnant women with Coronavirus Disease 2019. A systematic review. Travel medicine and infectious disease, 101919.

Oncel, M. Y., Akın, I. M., Kanburoglu, M. K., Tayman, C., Coskun, S., Narter, F., \& Koc, E. (2021). A multicenter study on epidemiological and clinical characteristics of 125 newborns born to women infected with COVID-19 by Turkish Neonatal Society. European journal of pediatrics, $180(3)$, $733-742$.

Papapanou, M., Papaioannou, M., Petta, A., Routsi, E., Farmaki, M., Vlahos, N., \& Siristatidis, C. (2021). Maternal and Neonatal Characteristics and Outcomes of COVID-19 in Pregnancy: An Overview of Systematic Reviews. International Journal of Environmental Research and Public Health, 18 (2), 596.

Pirjani, R., Hosseini, R., Soori, T., Rabiei, M., Hosseini, L., Abiri, A., \& Sepidarkish, M. (2020). Maternal and neonatal outcomes in COVID-19 infected pregnancies: a prospective cohort study. Journal of Travel Medicine, 27(7), taaa158.

Rasmussen, S. A., \& Jamieson, D. J. (2020). Coronavirus disease 2019 (COVID-19) and pregnancy: responding to a rapidly evolving situation. Obstetrics and gynecology.

Remaeus, K., Savchenko, J., Brismar Wendel, S., Brusell Gidlöf, S., Graner, S., Jones, E., \& Pettersson, K. (2020). Characteristics and short-term obstetric outcomes in a case series of 67 women test-positive for SARS-CoV-2 in Stockholm, Sweden. Acta Obstetricia et Gynecologica Scandinavica, 99(12), 16261631 .

Sattari, M., Bashirian, S., Masoumi, S. Z., Shayan, A., Jenabi, E., Ghelichkhani, S., \& Alimohammadi, S. (2020). Evaluating clinical course and risk factors of infection and demographic characteristics of pregnant women with COVID-19 in Hamadan Province, West of Iran. Journal of research in health sciences, 20(3), e00488.

Savasi, V. M., Parisi, F., Patanè, L., Ferrazzi, E., Frigerio, L., Pellegrino, A., \& Cetin, I. (2020). Clinical findings and disease severity in hospitalized pregnant women with coronavirus disease 2019 (COVID-19). Obstetrics \& Gynecology, 136(2), 252-258. 
Research, Society and Development, v. 10, n. 7, e16110716283, 2021

(CC BY 4.0) | ISSN 2525-3409 | DOI: http://dx.doi.org/10.33448/rsd-v10i7.16283

Sentilhes, L., De Marcillac, F., Jouffrieau, C., Kuhn, P., Thuet, V., Hansmann, Y., \& Deruelle, P. (2020). Coronavirus disease 2019 in pregnancy was associated with maternal morbidity and preterm birth. American Journal of Obstetrics and Gynecology, $223(6), 914-\mathrm{e} 1$.

Siston, A. M., Rasmussen, S. A., Honein, M. A., Fry, A. M., Seib, K., Callaghan, W. M., \& Pandemic H1N1 Influenza in Pregnancy Working Group. (2010). Pandemic 2009 influenza A (H1N1) virus illness among pregnant women in the United States. Jama, 303(15), 1517-1525.

Smith, V., Seo, D., Warty, R., Payne, O., Salih, M., Chin, K. L., \& Wallace, E. (2020). Maternal and neonatal outcomes associated with COVID-19 infection: A systematic review. Plos one, 15(6), e0234187.

Souza, M. T. D., Silva, M. D. D., \& Carvalho, R. D. (2010). Revisão integrativa: o que é e como fazer. Einstein (São Paulo), 8(1), $102-106$.

Turan, O., Hakim, A., Dashraath, P., Jeslyn, W. J. L., Wright, A., \& Abdul-Kadir, R. (2020). Clinical characteristics, prognostic factors, and maternal and neonatal outcomes of SARS-CoV-2 infection among hospitalized pregnant women: A systematic review. International Journal of Gynecology \& Obstetrics, 151(1), 7-16.

Twilhaar, E. S., Wade, R. M., De Kieviet, J. F., Van Goudoever, J. B., Van Elburg, R. M., \& Oosterlaan, J. (2018). Cognitive outcomes of children born extremely or very preterm since the 1990s and associated risk factors: a meta-analysis and meta-regression. JAMA pediatrics, 172(4), 361-367.

Vollmer, B., \& Stålnacke, J. (2019). Young adult motor, sensory and cognitive outcomes and longitudinal development after very and extremely preterm birth. Neuropediatrics, 50(4), 219-227.

Yan, J., Guo, J., Fan, C., Juan, J., Yu, X., Li, J., \& Yang, H. (2020). Coronavirus disease 2019 in pregnant women: a report based on 116 cases. American journal of obstetrics and gynecology, 223(1), 111-e1.

Yang, R., Mei, H., Zheng, T., Fu, Q., Zhang, Y., Buka, S., \& Zhou, A. (2020). Pregnant women with COVID-19 and risk of adverse birth outcomes and maternal-fetal vertical transmission: a population-based cohort study in Wuhan, China. BMC medicine, 18(1), 1-7.

Yee, J., Kim, W., Han, J. M., Yoon, H. Y., Lee, N., Lee, K. E., \& Gwak, H. S. (2020). Clinical manifestations and perinatal outcomes of pregnant women with COVID-19: a systematic review and meta-analysis. Scientific reports, 10(1), 1-7.

Zhu, N., Zhang, D., Wang, W., Li, X., Yang, B., Song, J., \& Tan, W. (2020). A novel coronavirus from patients with pneumonia in China, 2019. New England journal of medicine. 